Revista de Matemática: Teoría y Aplicaciones 4(1): 31-42 (1997)

\title{
COPRODUCTOS FIBRADOS DE MORFISMOS K-FINITOS
}

\author{
Osvaldo Acuña Ortega ${ }^{1}$
}

\begin{abstract}
Resumen
En éste artículo se estudian condiciones para que coproductos fibrados de morfismos $K$-finitos sean $K$-finitos
\end{abstract}

\begin{abstract}
In this paper we study conditions under which the push-out of morphisms $K$-finite are $K$-finite, and some related problems
\end{abstract}

\section{Introducción}

En el presente trabajo se estudian condiciones para que coproductos fibrados de morfismos $K$-finitos sean $K$-finitos. Se prueba que un morfismo $f: X \rightarrow Y$ es $K$-finito si y solo si los morfismos de su factorización epic-monic lo son también. Se prueba un teorema donde se dan varias condiciones equivalentes a la propiedad de $K$-finitud para monomorfismos.

Este artículo es la continuación del artículo Algunos aspectos de morfismos $K$-finitos [2], del mismo autor, y por tanto el presente trabajo sigue y utiliza las notaciones, definiciones y ciertos resultados de éste.

\section{Desarrollo}

Proposición 1. Sea $\mathcal{E}$ un topos y $f: X \rightarrow Y$ un morfismo. Si $f=i \circ q$, la factorización epic-monic de $f$ entonces $f$ es un morfismo $K$-finito si y solo si $i, q$ son ambos morfismos $K$-finitos.

Prueba. $(\Leftarrow) i, q$ son $K$-finitos entonces $f=i \circ q$ es $K$-finito por proposición 3 de [2]. $(\Rightarrow) \mathrm{Si}$

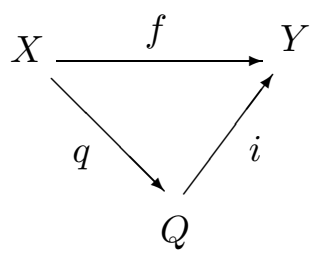

\footnotetext{
${ }^{1}$ Centro de Investigaciones en Matemáticas Puras y Aplicadas (Cimpa), Escuela de Matemática, Universidad de Costa Rica, 2060 San José, Costa Rica
} 
es el diagrama de factorización de $f$, con $q$ epic y $i$ monic. Entonces

$$
\models f^{-1}(i(s))=(i \circ q)^{-1}(i(s))=q^{-1}\left(i^{-1}(i(s))\right)=q^{-1}(s)
$$

y luego

$$
\models \forall s \in Q \quad\left(q^{-1}(s)=f^{-1}(i(s))\right)
$$

Como $f$ es un morfismo $K$-finito tenemos por Teorema 3.2.8 de [1] que

$$
\models \forall s \in Q\left(q^{-1}(s) \in K(X)\right)
$$

luego $q$ es un morfismo $K$-finito.

Por otro lado en $\mathcal{E} / X$ el soporte de $f: X \rightarrow Y$ es $i: Q \longmapsto Y$, como $f$ es $K$-finito entonces $i$ es $K$-finito en $\mathcal{E} / X$ por teorema 3.6 de [3].

Corolario 2. Si $f: X \rightarrow Y$ es un morfismo $K$-finito y si $Q$ es la imagen de $f$. Entonces $Q$ tiene complemento en $Y$.

Prueba. Como $f$ es $K$-finito entonces $i: Q \rightarrow Y$ es $K$-finito y por proposición 2 de [2] $Q$ tiene complemento en $Y$.

Teorema 3. Sea $i: I \rightarrow X$ un monomorfismo en un topos $\mathcal{E}$. Entonces las siguientes afirmaciones son equivalentes

(a) $i: I \rightarrow X$ tiene complemento

(b) $i: I \rightarrow X$ es un morfismo $K$-finito

(c) $p=<p_{1}, p_{2}>: X+X \rightarrow Q$ es $K$-finito si

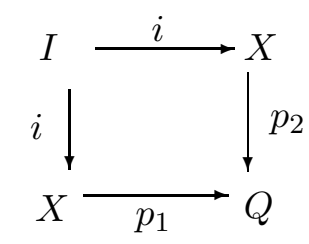

es un coproducto fibrado.

(d) $p_{1}, p_{2}: X \rightarrow Q$ son ambos $K$-finitos $\left(p_{1}, p_{2}\right.$ como en (c))

Prueba. $(\mathrm{a}) \Longleftrightarrow$ (b) proposición 2 de [2]. (a) $\Rightarrow$ (c) proposición 4 de [2]. (c) $\Longleftrightarrow$ (d) Si $i_{1}, i_{2}: X \rightarrow X+X$ son las dos inclusiones del coproducto, tenemos que $p_{1}=p \circ i_{1}$, $p_{2}=p \circ i_{2}$. Dado que $i_{1}, i_{2}, p$ son morfismos $K$-finitos por proposición 3 de [2] $p_{1}, p_{2}$ son $K$-finitos.
(d) $\Rightarrow$ (b) Como 


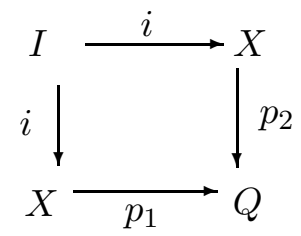

es también un producto fibrado, siendo $p_{2} K$-finito entonces $i$ es $K$-finito.

Lema 4. Sea $f: Y \rightarrow X$ un morfismo y $Y \stackrel{q}{\rightarrow} I \stackrel{i}{\rightarrow} X$ su factorización epic-monic. Entonces



es un coproducto fibrado si y solo si

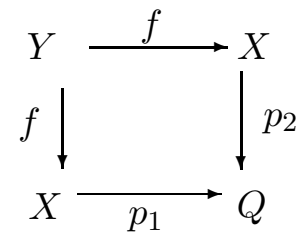

es un coproducto fibrado.

Prueba. $(\Rightarrow)$ Sean $g_{1}, g_{2}: X \rightarrow Z$ tal que $g_{1} \circ f=g_{2} \circ f$. Considere el siguiente digrama conmutativo.

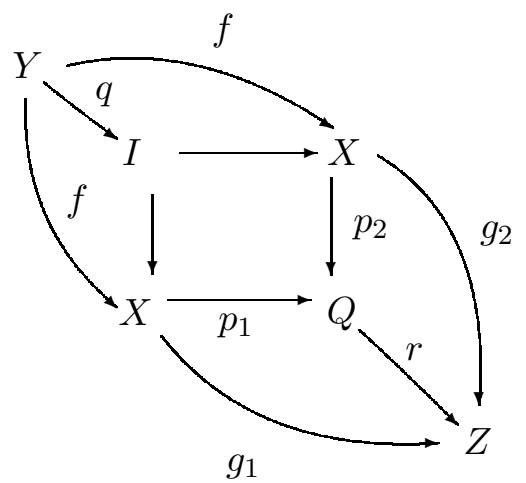

Tenemos que $g_{1} \circ i \circ q=g_{2} \circ i \circ q$, como $q$ es epic entonces $g_{1} \circ i=g_{2} \circ i$. Por lo tanto existe un único morfismo $r: Q \rightarrow Z$ tal que $r \circ p_{1}=g_{1}$ y $r \circ p_{2}=g_{2}$. En consecuencia 




es un producto fibrado.

$(\Leftarrow)$ trivial.

Teorema 5. Sea $f: X \rightarrow Y$ un morfismo en un topos $\mathcal{E}$. Si $f$ es $K$-finito y

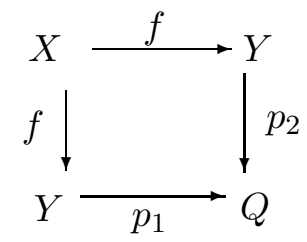

es un coproducto fibrado entonces $p_{1}, p_{2}$ son $K$-finitos.

Reciprocamente si $p_{1}$ o $p_{2}$ son $K$-finitos entonces la imagen $I$ de $f$ en $Y$ tiene complemento o equivalentemente la inclusión $i: I \rightarrow Y$ es $K$-finita.

Prueba. Si

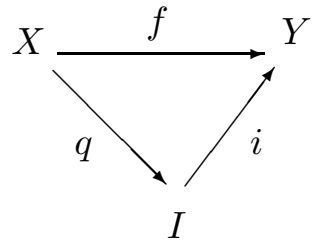

es la factorización epic-monic entonces como $f$ es $K$-finito por proposición $1, i: I \rightarrow Y$ es $K$-finito y por el lema 4 y (d) del teorema $3 p_{1}, p_{2}$ son $K$-finitos. Recíprocamente, si $p_{1}$, $p_{2}$ un $k$-finito como

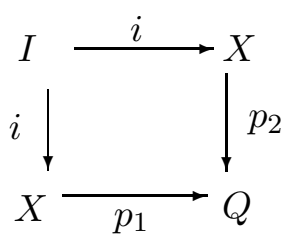

es un coproducto fibrado por lema 4, dado que $i$ es monic, éste diagrama debe ser también un producto fibrado como $p_{1}$ o $p_{2}$ son morfismos $K$-finitos, ésto implica que $i: I \rightarrow X$ es $K$-finito por corolario 9.17 de [4].

Definición 1. Sean $g: X \rightarrow Z$ y $f: X \rightarrow Y$ dos morfismos en un topos $\mathcal{E}$. Si

$$
i_{1}: Y \rightarrow Y+Z \longleftarrow Z: i_{2}
$$

es un diagrama coproducto. Sean

$$
R^{*}=\cap\{R \subseteq(Y+Z) \times(Y+Z) / R \text { es un relación de }
$$


equivalencia $\left.\wedge\left\{\left(i_{1}(f(x)), i_{2}(g(x))\right) / x \in X\right\} \subseteq R\right\}$

$Q=(Y+Z) / R^{*}, p_{1}=\pi \circ i_{1}: Y \rightarrow Q$ y $p_{2}=\pi \circ i_{2}: Z \rightarrow Q$ donde $\pi: Y+Z \rightarrow Q$ es el morfismo cociente.

Proposición 6. Con la notación de la definición anterior tenemos que

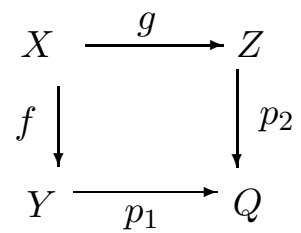

es un coproducto fibrado.

Prueba. Como $\pi \circ i_{1} \circ f=\pi \circ i_{2} \circ g$, entonces $p_{1} \circ f=p_{2} \circ f$ y por lo tanto $(*)$ conmuta. Considere el siguiente diagrama conmutativo:

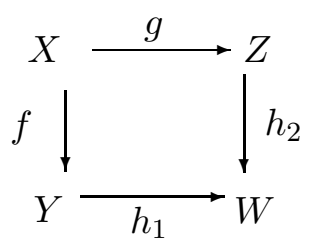

Sea $h: Y+Z \rightarrow W$ tal que $h \circ i_{1}=h_{1}, h \circ i_{2}=h_{2}$ y $R_{h}$ la relación de equivalencia definida por

$$
\models(x, y) \in R_{h} \Longleftrightarrow h(x)=h(y)
$$

Como $h_{1} \circ f=h_{2} \circ g$ tenemos $h \circ i_{1} \circ f=h \circ i_{2} \circ g$ entonces

$$
\begin{aligned}
\models x \in X & \Rightarrow h\left(i_{1}(f(x))\right)=h\left(i_{2}(g(x))\right) \\
& \Rightarrow\left(i_{1}(f(x)), i_{2}(g(x))\right) \in R_{h}
\end{aligned}
$$

entonces

$$
\models\left\{\left(i_{1}(f(x)), i_{2}(g(x))\right) / x \in X\right\} \subseteq R_{h}
$$

por lo tanto $R^{*} \subseteq R_{h}$.

Sea $j: Q \rightarrow Z$ definida tal que $=j([s])=h(x)$ donde $[s]$ es la clase de equivalencia de $x$ con $x \in Y+Z$.

Debemos probar que $j$ esté bien definida:

$$
\begin{aligned}
\models[s]=\left[s^{\prime}\right] & \Rightarrow\left(s, s^{\prime}\right) \in R^{*} \\
& \Rightarrow\left(s, s^{\prime}\right) \in R_{h} \\
& \Rightarrow h(s)=h\left(s^{\prime}\right)
\end{aligned}
$$

Queremos probar que $j$ es un morfismo, único tal que el siguiente diagrama conmuta 


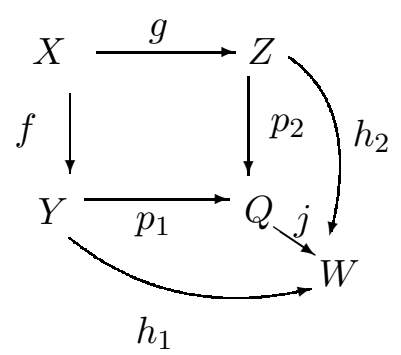

Por definición de $j$ tenemos $j \circ \pi=h$ y entonces

$$
\begin{aligned}
& j \circ p_{1}=j \circ \pi \circ i_{1}=h \circ i_{1}=h_{1} \\
& j \circ p_{2}=j \circ \pi \circ i_{2}=h \circ i_{2}=h_{2}
\end{aligned}
$$

por lo tanto $j \circ p_{1}=h_{1}$ y $j \circ p_{2}=h_{2}$.

Por otra lado suponga que existe otro morfismo $K: Q \rightarrow W$ tal que $k \circ p_{1}=h_{1} \mathrm{y}$ $k \circ p_{2}=h_{2}$ entonces $k \circ \pi \circ i_{1}=h_{2}$ y $k \circ \pi \circ i_{2}=h_{1}$ y por definición del coproducto $Y+Z$ tenemos que $k \circ \pi=h$, del coproducto $Y+Z$ tenemos que $k \circ \pi=h$, así se obtiene que $j \circ \pi=k \circ \pi$, como $\pi$ es un epímorfismo se tiene que $j=k$.

Definición 2. Sean $f: X \rightarrow Y$ y $g: X \rightarrow Z$, dos morfismos, denote por $R$ el subobjeto de $(Y+Z) \times(Y+Z)$ definido por la unión siguiente:

$$
\begin{gathered}
\left\{i_{1}(f(x)), i_{1}(f(\bar{x})) / x, \bar{x} \in X \wedge g(x)=g(\bar{x})\right\} \cup \Delta_{Y+Z} \cup \\
\left\{\left(i_{1},(f(x)), i_{2}(g(x))\right) / x \in X\right\} \cup\left\{\left(i_{2}(g(x)), i_{1}(f(x)) / x \in X\right\}\right.
\end{gathered}
$$

donde $Y \stackrel{i_{1}}{\rightarrow} Y+Z \stackrel{i_{2}}{\longleftarrow} Z$ es un diagrama coproducto.

Proposición 7. Si $f$ es un monomorfismo entonces $R=R^{*}$, en particular $R$ es una relación de equivalencia.

Prueba. Primero probamos que $R$ es una relación de equivalencia

(i) $R$ es reflexiva ya que $\Delta_{Y+Z} \subseteq R$

(ii) $R$ es simétrica:

$$
\begin{aligned}
\models=(t, s) \in R \Rightarrow & \left(\exists_{x, \bar{x} \in X}\left(t=i_{1}(f(x)) \wedge s=i_{1}(f(\bar{x})) \wedge g(x)=g(\bar{x})\right)\right) \vee t=s \\
& \vee \exists_{x \in X}\left(t=i_{1}(f(x)) \wedge s=i_{2}(g(x))\right) \vee \exists_{x \in X}\left(t=i_{2}(g(x))\right. \\
& \left.\wedge s=i_{1}(f(x))\right) \\
\Rightarrow \quad & (s, t) \in R \vee(s, t) \in R \vee(s, t) \in R \vee(s, t) \in R \\
\Rightarrow & (s, t) \in R
\end{aligned}
$$

(iii) $R$ es transitiva. 


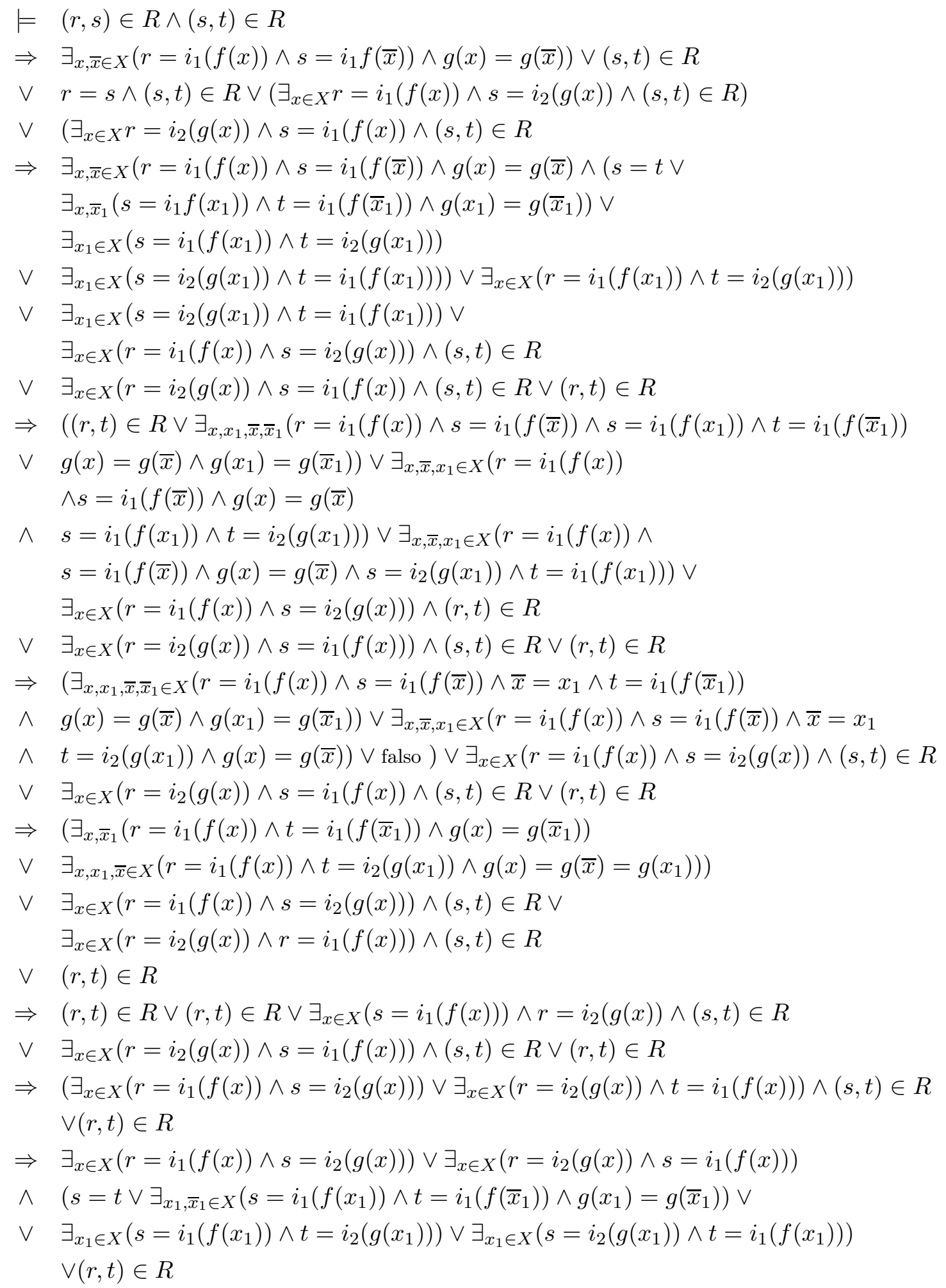




$$
\begin{aligned}
\Rightarrow & (r, t) \in R \vee \text { falso } \vee \exists_{x, x_{1}, \bar{x}_{1} \in X}\left(r=i_{2}(g(x)) \wedge s=i_{1}(f(x)) \wedge s=i_{1}(f(x))\right. \\
\wedge & \left.t=i_{1}\left(f\left(\bar{x}_{1}\right)\right) \wedge g\left(x_{1}\right)=g\left(\bar{x}_{1}\right)\right) \vee \text { falso } \vee \exists_{x, x_{1} \in X}\left(r=i_{2}(g(x)) \wedge s=i_{1}(f(x))\right. \\
\wedge & \left.s=i_{1}\left(f\left(x_{1}\right)\right) \wedge t=i_{2}\left(g\left(x_{1}\right)\right)\right) \vee \exists_{x, x_{1} \in X}\left(r=i_{1}(f(x)) \wedge s=i_{2}(g(x))\right. \\
\wedge & \left.s=i_{2}\left(g\left(x_{1}\right)\right) \wedge t=i_{1}\left(f\left(x_{1}\right)\right)\right) \vee \text { falso } \vee(r, t) \in R \\
\Rightarrow \quad & \exists_{x, x_{1}, \bar{x}_{1} \in X}\left(r=i_{2}(g(x)) \wedge x=x_{1} \wedge t=i_{1}\left(f\left(\bar{x}_{1}\right) \wedge g\left(x_{1}\right)=g\left(\bar{x}_{1}\right)\right) \vee\right. \\
\vee \quad & \exists_{x, x_{1} \in X}\left(r=i_{2}(g(x)) \wedge x=x_{1} \wedge t=i_{2}\left(g\left(x_{1}\right)\right)\right) \vee \exists_{x, x_{1} \in X}\left(r=i_{1}(f(x)) \wedge\right. \\
& \left.x=x_{1} \wedge t=i_{1}\left(f\left(x_{1}\right)\right)\right) \vee(r, t) \in R \\
\Rightarrow & \exists_{x_{1} \in X}\left(r=i_{2}\left(g\left(\bar{x}_{1}\right)\right) \wedge t=i_{1}\left(f\left(\bar{x}_{1}\right)\right)\right) \vee \exists_{x \in X}\left(r=i_{2}(g(x)) \wedge t=i_{2}(g(x))\right) \\
& \vee \exists_{x \in X}\left(r=i_{1}(f(x)) \wedge t=i_{1}(f(x))\right) \vee(r, t) \in R \\
\Rightarrow \quad & (r, t) \in R \vee r=t \vee r=t \vee(r, t) \in R \\
\Rightarrow \quad & (r, t) \in R
\end{aligned}
$$

Por lo tanto $R$ es transitiva y entonces es una relación de equivalencia. Por otro lado como

$$
\left\{\left(i_{1}(f(x)), i_{2}(g(x))\right) / x \in X\right\} \subseteq R,
$$

siendo $R$ una relación de equivalencia, tenemos que $R^{*} \subseteq R$.

Si $R^{\prime}$ es cualquier relación de equivalencia que contiene

$$
\left\{\left(i_{1}(f(x)), i_{2}(g(x))\right) / x \in X\right\}
$$

entonces $R^{\prime} \supseteq\left\{\left(i_{2}(g(x)), i_{1}(f(x))\right) / x \in X\right\}$. Por otro lado tenemos que

$$
\begin{aligned}
\models & x, \bar{x} \in X \wedge g(x)=g(\bar{x}) \\
\Rightarrow & \left(i_{1}(f(x)), i_{2}(g(x)) \in R^{\prime} \wedge\left(i_{1}(f(\bar{x})), i_{2}(g(\bar{x})) \in R^{\prime}\right.\right. \\
& \wedge g(x)=g(\bar{x}) \\
\Rightarrow & \left(i_{1}(f(x)), i_{2}(g(x)) \in R^{\prime} \wedge\left(i_{2}(g(x)), i_{1}(f(\bar{x}))\right) \in R^{\prime}\right. \\
\Rightarrow & \left(i_{1}(f(x)), i_{1}(f(\bar{x}))\right) \in R^{\prime} \\
\Rightarrow & \left\{i_{1}(f(x)), i_{1}(f(\bar{x})) / x, \bar{x} \in X \wedge g(x)=g(\bar{x})\right\} \subseteq R^{\prime}
\end{aligned}
$$

Por lo tanto $R \subseteq R^{\prime}$ y entonces $R \subseteq R^{*}$ y $R=R^{*}$.

Proposición 8. En el siguiente coproducto fibrado

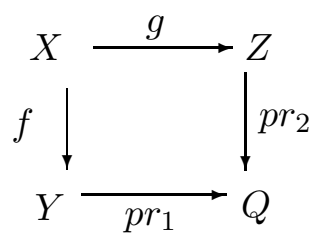

tenemos que si $f$ es un monomorfismo, entonces $f$ es un morfismo $K$-finito si y sólo si $p r_{2}$ es un morfismo $K$-finito. En particular la imagen de $f$ es $Y$ tiene complemento si y sólo si la imagen de $p r_{2}$ en $Q$ tiene complemento. 
Prueba. Es conocido que si $f$ es un monomorfismo entonces $\left(^{*}\right)$ es también un producto fibrado y $p r_{2}$ es un monomorfismos. En particular si $p r_{2}$ es $K$-finito, entonces $f$ es también $K$-finito ya que los funtores imagen inversa de morfismos geométricos preservan objetos $K$-finitos.

Recíprocamente suponga que $f$ es un monomorfismo $K$-finito entonces si $X^{\prime}$ es la imagen de $f, X^{\prime}$ tiene complemento $X^{\prime \prime}$ en $Y$. Sea $Z^{\prime}$ es la imagen de $p r_{2}$ en $Q$ y $Z^{\prime \prime}$ la imagen del morfismo

$$
p r_{1} / X^{\prime \prime}: X^{\prime \prime} \rightarrow Q
$$

Queremos probar que $Z^{\prime \prime}$ es el complemento de $Z^{\prime}$ y como $p r_{2}$ es monic, esto implicaría que $p r_{2}$ es un morfismo $K$-finito.

Primero probemos que

$$
Z^{\prime} \cap Z^{\prime \prime}=\emptyset
$$

$$
\begin{aligned}
& =q \in Z^{\prime} \cap Z^{\prime \prime} \\
& \Rightarrow \exists_{z \in Z} q=\operatorname{pr}_{2}(z) \wedge \exists_{y \in Y}\left(q=p r_{1}(y) \wedge y \in X^{\prime \prime}\right) \\
& \Rightarrow \exists_{z \in Z, y \in Y}\left(\operatorname{pr}_{2}(z)=\operatorname{pr}_{1}(y) \wedge y \in X^{\prime \prime}\right) \\
& \Rightarrow \exists_{z \in Z, y \in Y}\left(\pi\left(i_{2}(z)\right)=\pi\left(i_{1}(y)\right) \wedge y \in X^{\prime \prime}\right) \\
& \Rightarrow \exists_{z \in Z, y \in Y}\left(\left(i_{2}(z), i_{1}(y)\right) \in R \wedge y \in X^{\prime \prime}\right) \\
& \Rightarrow \exists_{z \in Z, y \in Y}\left(\text { falso } \vee \text { falso } \vee \text { falso } \vee \exists_{x \in X} z=g(x) \wedge i_{1}(f(x))=i_{1}(y) \wedge y \in X^{\prime \prime}\right) \\
& \Rightarrow \exists_{z \in Z, y \in Y}\left(y \in X^{\prime} \wedge y \in X^{\prime \prime}\right) \\
& \Rightarrow \exists_{z \in Z, y \in Y}(\text { falso }) \\
& \Rightarrow \text { falso }
\end{aligned}
$$

Por lo tanto $=q \in Z^{\prime} \cap Z^{\prime \prime} \Rightarrow$ falso. Luego $Z^{\prime} \cap Z^{\prime \prime}=\emptyset$

Probemos ahora que

$$
Z^{\prime} \cup Z^{\prime \prime}=Q
$$

$$
\begin{aligned}
\models & q \in Q \\
\Rightarrow & \exists_{y \in Y} q=\pi\left(i_{1}(y)\right) \vee \exists_{z \in Z} q=\pi\left(i_{2}(z)\right) \\
\Rightarrow & \exists_{x \in X} q=\pi\left(i_{1}(f(x)) \vee \exists_{x^{\prime \prime} \in X^{\prime \prime}} q=\pi\left(i_{1}\left(x^{\prime \prime}\right)\right) \vee \exists_{z \in Z} q=\pi\left(i_{2}(z)\right)\right. \\
\Rightarrow & \exists_{x \in X} q=p \pi_{1}(f(x)) \vee q \in Z^{\prime \prime} \vee q \in Z^{\prime} \\
& \left(\operatorname{como} p \pi_{1}(f(x))=p r_{2}(g(x))\right) \\
\Rightarrow & \exists_{x \in X} q=p r_{2}(g(x)) \vee q \in Z^{\prime \prime} \vee q \in Z^{\prime} \\
\Rightarrow & q \in Z^{\prime} \vee q \in Z^{\prime \prime} \vee q \in Z^{\prime} \\
\Rightarrow & q \in Z^{\prime} \vee q \in Z^{\prime \prime} \\
\Rightarrow & q \in Z^{\prime} \cup Z^{\prime \prime}
\end{aligned}
$$

Por lo tanto $Q=Z^{\prime} \cup Z^{\prime \prime}$ y $Z^{\prime \prime}$ es el complemento de $Z^{\prime}$ en $Q$, que es lo que queríamos probar.

Corolario 9. Si en el siguiente coproducto fibrado 




$f$ es un monomorfismo $K$-finito y $g$ es un morfismo $K$-finito entonces $p r_{1}$ es un morfismo $K$-finito.

Prueba. Si $g$ es un monomorfismo por la proposición anterior $p r_{1}$ es $K$-finito.

Suponga que $g$ es un epimorfismo, entonces $p r_{1}$ es un epimorfismo también. Sea $Y^{\prime}$ la imagen de $f$ en $Y$ es $Y^{\prime \prime}$ su complemento, entonces

$$
\begin{aligned}
& \models \bar{y} \in Y^{\prime \prime} \wedge y \in\left(p r_{1}\right)^{-1}\left(p r_{1}(\bar{y})\right) \\
& \Rightarrow \operatorname{pr}_{1}(y)=p r_{1}(\bar{y}) \wedge \bar{y} \in Y^{\prime \prime} \\
& \Rightarrow \quad\left(i_{1}(y), i_{1}(\bar{y})\right) \in R \wedge \bar{y} \in Y^{\prime \prime} \\
& \Rightarrow i_{1}(y)=i_{1}(\bar{y}) \vee \text { falso } \vee \text { falso } \vee \text { falso } \\
& \Rightarrow y=\bar{y} \\
& \Rightarrow y \in\{\bar{y}\}
\end{aligned}
$$

Por lo tanto

$$
\begin{aligned}
& \models \bar{y} \in Y^{\prime \prime} \\
& \Rightarrow \quad \forall_{y \in Y}\left(y \in\left(p r_{1}\right)^{-1}\left(p r_{1}(\bar{y})\right) \Rightarrow y \in\{\bar{y}\}\right) \\
& \Rightarrow \quad\left(p r_{1}\right)^{-1}\left(\operatorname{pr}_{1}(\bar{y})\right) \subseteq\{\bar{y}\}
\end{aligned}
$$

como $\models\{\bar{y}\} \subseteq\left(p r_{1}\right)^{-1}\left(p r_{1}(\bar{y})\right)$ entonces

$$
\begin{aligned}
\models \bar{y} \in Y^{\prime \prime} & \Rightarrow\left(p r_{1}\right)^{-1}\left(p r_{1}(\bar{y})\right)=\{\bar{y}\} \\
& \Rightarrow\left(p r_{1}\right)^{-1}\left(p r_{1}(\bar{y})\right) \in K(Y)
\end{aligned}
$$

luego

$$
\models \bar{y} \in Y^{\prime \prime} \Rightarrow\left(p r_{1}\right)^{-1}\left(p r_{1}(y)\right) \in K(Y)
$$

Por otro lado

$$
\begin{aligned}
= & \bar{x} \in X \wedge y \in\left(p r_{1}\right)^{-1}\left(\operatorname{pr}_{1}(f(\bar{x}))\right) \\
\Rightarrow & \operatorname{pr}_{1}(y)=p r_{1}(f(\bar{x})) \\
\Rightarrow & \left(i_{1}(y), i_{1}(f(\bar{x})) \in R\right. \\
\Rightarrow & i_{1}(y)=i_{1}(f(x)) \vee \exists_{x_{1}, x_{2} \in X}\left(i_{1}(y)=i_{1}\left(f\left(x_{1}\right)\right) \wedge i_{1}(f(\bar{x}))=\right. \\
& i_{1}\left(f\left(x_{2}\right)\right) \wedge g\left(x_{1}\right)=g\left(x_{2}\right) \vee \text { falso } \vee \text { falso } \\
\Rightarrow & y=f(\bar{x}) \vee \exists_{x_{1}, x_{2} \in X}\left(y=f\left(x_{1}\right) \wedge \bar{x}=x_{2} \wedge g\left(x_{1}\right)=g\left(x_{2}\right)\right) \\
\Rightarrow & y \in\{f(\bar{x})\} \vee \exists_{x_{1} \in X}\left(y=f\left(x_{1}\right) \wedge g\left(x_{1}\right)=g(\bar{x})\right) \\
\Rightarrow & y \in\{f(\bar{x})\} \vee \exists_{x_{1} \in X}\left(y=f\left(x_{1}\right) \wedge x_{1} \in g^{-1}(g(\bar{x}))\right.
\end{aligned}
$$




$$
\begin{gathered}
\Rightarrow \quad y \in\{f(\bar{x})\} \vee y \in f\left(g^{-1}(g(\bar{x}))\right) \\
\quad \text { como } \models f(\bar{x}) \in f\left(g^{-1}(g(\bar{x}))\right) \\
\Rightarrow \quad y \in f\left(g^{-1}(g(\bar{x}))\right)
\end{gathered}
$$

Así tenemos que

$$
\vDash \bar{x} \in X \Rightarrow \forall_{y \in Y}\left(y \in\left(p r_{1}\right)^{-1}\left(p r_{1}(f(\bar{x})) \Rightarrow y \in f\left(g^{-1}(g(\bar{x}))\right)\right)\right.
$$

es decir

$$
\models \bar{x} \in X \Rightarrow\left(p r_{1}\right)^{-1}\left(p r_{1}(f(\bar{x})) \subseteq f\left(g^{-1}(g(\bar{x}))\right)\right)
$$

Tenemos también que

$$
\begin{aligned}
& =\quad z \in f\left(g^{-1}(g(\bar{x}))\right) \\
& \Rightarrow \quad \exists_{x \in X}(z=f(x) \wedge g(x)=g(\bar{x})) \\
& \Rightarrow \quad\left(i_{1}(z), i_{1}(f(\bar{x}))\right) \in R \\
& \Rightarrow \pi \circ i_{1}(z)=\pi \circ i_{1}(f(\bar{x})) \\
& \Rightarrow \operatorname{pr}_{1}(z)=\operatorname{pr}_{1}(f(\bar{x})) \\
& \Rightarrow \quad z \in\left(p r_{1}\right)^{-1}\left(p r_{1}(f(\bar{x}))\right)
\end{aligned}
$$

luego

$$
\models f\left(g^{-1}(g(x))\right) \subseteq\left(p r_{1}\right)^{-1}\left(p r_{1}(f(\bar{x}))\right)
$$

entonces

$$
\models \bar{x} \in X \Rightarrow\left(p r_{1}\right)^{-1}\left(p r_{1}(f(\bar{x}))\right)=f\left(g^{-1}(g(\bar{x})) \quad .\right.
$$

Dado que $g$ es un morfismo $K$-finito sabemos que $\models \forall \bar{x} \in X g^{-1}(g(\bar{x})) \in K(X)$ y como $f(K(X)) \subseteq K(Y)$ esto implica

$$
\models \bar{x} \in X \Rightarrow\left(p r_{1}\right)^{-1}\left(p r_{1}(f(\bar{x}))\right) \in K(Y)
$$

y entonces

$$
\begin{aligned}
\models \bar{y} \in Y^{\prime} & \Rightarrow \exists_{\bar{x} \in X} \bar{y}=f(\bar{x}) \\
& \Rightarrow \exists_{\bar{x} \in X} \bar{y}=f(x) \wedge\left(p r_{1}\right)^{-1}\left(p r_{1}(f(\bar{x}))\right) \in K(Y) \\
& \Rightarrow \exists_{\bar{x} \in X}\left(p r_{1}\right)^{-1}\left(p r_{1}(\bar{y})\right) \in K(Y) \\
& \Rightarrow\left(p r_{1}\right)^{-1}\left(p r_{1}(\bar{y})\right) \in K(Y)
\end{aligned}
$$

es decir

$$
\models y \in Y^{\prime} \Rightarrow\left(p r_{1}\right)^{-1}\left(p r_{1}(\bar{y})\right) \in K(Y) .
$$

Como $Y=Y^{\prime} \cup Y^{\prime \prime}$ obtenemos

$$
\models y \in Y \Rightarrow\left(p r_{1}\right)^{-1}\left(p r_{1}(y)\right) \in K(Y)
$$

que es equivalente a decir que $p r_{1}$ es un morfismo $K$-finito ya que $p r_{1}: Y \rightarrow Q$ es un epimorfismo. 
Si $g$ es un morfismo general y $g=i \circ q$ es la factorización epic-monic, considere el diagrama

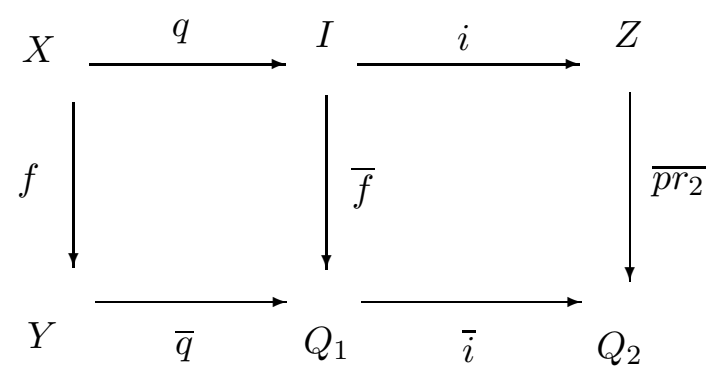

donde los dos cuadrados son coproductos fibrados. Por lo tanto el rectángulo formados por estos cuadrados es también un coproducto de fibrado y entonces $\bar{i} \circ \bar{q}$ es isomorfo a

$p r_{1}$. Por los argumentos anteriores $\bar{i}, \bar{q}$ un morfismo $K$-finito lo que implica que $\bar{i} \circ \bar{q}$ es $K$-finito y entonces $p r_{1}$ es $K$-finito.

\section{Referencias}

[1] Acuña Ortega, O. (1977) Finiteness in Topoi. Diseertation, Wesleyan University, Middletown, Connecticut.

[2] Acuña Ortega, O. "Algunos aspectos de morfismos $K$-finitos en un topos elemental", por aparecer.

[3] Kock, A.; Lecouturuier, P.; Mikkelsen, C.J. (1975) "Some topos-theoretic concepts of finiteness", Model Theory and Topoi, Springer Lectures Notes in Math. 445: 209-283.

[4] Johnstone, P.T. (1977) Topos Theory. L.M.S Mathematical Monographs 10, Academic Press, New York. 